\title{
Betacellulin (BTC) Biases the EGFR To Dimerize with ErbB3몽
}

\author{
Jamie S. Rush, Joanne L. Peterson, ${ }^{1}$ and Brian P. Ceresa \\ Departments of Pharmacology and Toxicology (J.S.R., J.L.P., B.P.C.) and Visual Science (B.P.C.), University of Louisville, \\ Louisville, Kentucky
}

Received July 10, 2018; accepted September 17, 2018

\begin{abstract}
There are 13 known endogenous ligands for the epidermal growth factor receptor (EGFR) and its closely related ErbB receptor family members. We previously reported that betacelIulin (BTC) is more efficacious than epidermal growth factor (EGF) in mediating corneal wound healing, although the molecular basis for this difference was unknown. For the most part, differences between ligands can be attributed to variability in binding properties, such as the unique rate of association and dissociation, $\mathrm{pH}$ sensitivity, and selective binding to individual ErbB family members of each ligand. However, this was not the case for BTC. Despite being better at promoting wound healing via enhanced cell migration, BTC has reduced receptor affinity
\end{abstract}

and weaker induction of EGFR phosphorylation. These data indicate that the response of BTC is not due to enhanced affinity or kinase activity. Receptor phosphorylation and proximity ligation assays indicate that BTC treatment significantly increases ErbB3 phosphorylation and EGFR-ErbB3 heterodimers when compared with EGF treatment. We observed that EGFRErbB3 heterodimers contribute to cell migration, because the addition of an ErbB3 antagonist (MM-121) or RNA interferencemediated knockdown of ErbB3 attenuates BTC-stimulated cell migration compared with EGF. Thus, we demonstrate that, despite both ligands binding to the EGFR, BTC biases the EGFR to dimerize with ErbB3 to regulate the biologic response.

\section{Introduction}

The ErbB family of receptor tyrosine kinases (RTKs) have well-established roles in developmental biology, tissue homeostasis, and cancer biology (Wieduwilt and Moasser, 2008; Chen et al., 2016). All four epidermal growth factor (EGF) receptor (EGFR) family members (ErbB1, ErbB2, ErbB3, and ErbB4) share a number of structural and functional features, including size, the transmembrane orientation of the protein, and the mechanism of activation. The activation of ErbB receptors begins with ligand binding that induces receptor dimerization, transphosphorylation of cytoplasmic tyrosines, and docking of downstream effectors to those phosphotyrosines. These activated effectors induce biochemical changes that lead to modifications in cell biology. Each ErbB family member is unique in its activating ligands, degree of kinase activity, and cadre of downstream effectors. These features confer receptor-specific biochemical signals, which regulate the resulting cell biology.

Receptor-specific ligands initiate ErbB RTK signaling. There are 13 known ligands for the ErbB family of proteins, each encoded by a distinct gene (Pathak et al., 1995). These ligands differ in their tissue distribution as well as their rates

This work was supported by National Institutes of Health Grants [1R01EY021497] (B.P.C.) and [R21EY027032] (B.P.C.).

${ }^{1}$ Current affiliation: Arkansas College of Osteopathic Medicine, Fort Smith, Arkansas.

https://doi.org/10.1124/mol.118.113399.

[ This article has supplemental material available at molpharm. aspetjournals.org. of association and dissociation to each receptor. Not only do ligands drive the specificity of receptor-effector interactions, but also the duration and magnitude of effector response through differences in membrane trafficking (Wiley, 2003; Wang and Hung, 2012). Ultimately, the ligand-specific mechanisms influence the cellular and physiologic responses.

Despite the appreciation that ligands can induce receptorspecific signaling events, the molecular basis for these differences are not always clear due to the intrinsic barriers to ligand analysis. Knockdown of the EGFR results in embryonic lethality in mice, or death shortly after birth, highlighting its role in embryonic development (Threadgill et al., 1995). In contrast, mice engineered to individually knock out EGF (Luetteke et al., 1999), transforming growth factor- $\alpha$ (Mann et al., 1993), epigen (Dahlhoff et al., 2013), heparin-binding EGF (Jackson et al., 2003), betacellulin (BTC) (Jackson et al., 2003), or amphiregulin (Luetteke et al., 1999) reveal no lethal ligand-specific phenotypes, and all mice were viable and fertile. More subtle phenotypes include minor defects, such as altered epithelial tissue homeostasis, mammary tissue development, or a "wavy" phenotype of the hair (Ceresa et al., 2016). The distinct knockout phenotypes indicate ligand-specific roles in tissue development and homeostasis. The absence of lethality when a single ligand is knocked out is consistent with functional redundancy among the ligands for the most critical functions of the EGFR. Although the overlapping roles of the ligands are likely beneficial to animals, analysis of the physiologic contributions of individual

ABBREVIATIONS: BTC, betacellulin; EGF, epidermal growth factor; EGFR, epidermal growth factor receptor; IP, immunoprecipitation; MM-121, ErbB3 antagonist/seribantumab; NRG4, neuregulin 4; PLA, proximity ligation assay; RTK, receptor tyrosine kinase; shRNA, short hairpin RNA; siRNA, small interfering RNA. 
ligands is difficult. To circumvent the limitations of in vivo analysis, we, like many others, have turned to cell biology and biochemical assays to understand ligand-specific signaling.

Among the endogenous EGFR ligands, BTC is one of the most poorly understood. It was first identified as a secreted growth factor from pancreatic $\beta$-cell insulinomas (Sasada et al., 1993; Shing et al., 1993) and has been implicated in a number of physiologic processes, including $\beta$-cell proliferation (Li et al., 2001), keratinocyte proliferation (Schneider et al., 2008b), and angiogenesis (Schneider et al., 2008a). In endometrial, liver, and pancreatic cancers, BTC levels are elevated (Yokoyama et al., 1995; Srinivasan et al., 1999; Moon et al., 2006). BTC is a dual-specificity ligand that binds both EGFR and ErbB4 (Singh and Coffey, 2014; Singh et al., 2016). It has been argued that BTC also binds ErbB3 (López-Torrejón et al., 2002). The most compelling evidence for this comes from 32D myeloid progenitor cells that lack endogenous ErbB RTKs. When ErbB2 and ErbB3 are exogenously expressed, there is ErbB2 and ErbB3 tyrosine phosphorylation and increased ${ }^{3} \mathrm{H}$-thymidine incorporation (DNA synthesis) (Alimandi et al., 1997). However, a more systematic analysis used ErbB RTKs expressed individually or in pairs; these studies demonstrated that measurable binding of BTC only occurs when EGFR and ErbB4 are expressed (Jones et al., 1999).

BTC-null mice are viable and exhibit no overt phenotypes or problems with fertility; however, the life span of these mice is reduced (Jackson et al., 2003). Overexpression of BTC causes abnormal hair follicle development (Schneider et al., 2008a), increased glucose tolerance (Dahlhoff et al., 2009), and altered corneal development (Schneider et al., 2005). In corneal epithelial cells, BTC is more efficacious than EGF in mediating wound healing (Peterson et al., 2014). Despite evidence that BTC enhances wound healing under both physiologic and pathologic conditions, the molecular mechanism by which BTC enhances this process is unclear. These data illustrate the longstanding, inherent confusion regarding BTC-mediated signaling, and the need for a unifying model.

In this study, we found that BTC is a better mediator of cell migration than EGF, despite a lower affinity for and reduced phosphorylation of the EGFR (Watanabe et al., 1994). The reduced receptor phosphorylation is due to the formation of heterodimers in the EGFR with the kinase-impaired ErbB3. Importantly, BTC promotes ErbB3 phosphorylation in the absence of ErbB2. Proximity ligation assays (PLAs) directly reveal the formation of EGFR-ErbB3 heterodimers. When ErbB3 signaling is antagonized or depleted using ErbB3 antibody MM-121 (Schoeberl et al., 2009) or small interfering RNA (siRNA), cell migration is reduced more in response to BTC than to EGF, further indicating that BTC mediates migration through ErbB3. Together, these data provide a new model in which a ligand can bias dimerization partners of the EGFR and affect cell physiology.

\section{Materials and Methods}

Cell Lines. hTCEpi cells were obtained from Geron Corp. (Menlo Park, CA). Human corneal epithelial cells were immortalized by the stable transfection of human telomerase reverse transcriptase (Robertson et al., 2005). Cells were grown in growth media (Defined Keratinocyte with growth supplement; Invitrogen, Carlsbad, CA) at $37^{\circ} \mathrm{C}$ and were maintained at $5 \% \mathrm{CO}_{2}$. MDA-MB-468 cells were acquired from the American Type Culture Collection (Manassas, VA). Cells were maintained in growth media (Dulbecco's modified Eagle's medium) supplemented with $10 \%$ fetal bovine serum, $1 \%$ penicillin, $1 \%$ streptomycin, and $2 \mathrm{mM}$ glutamine, all acquired from Life Technologies (Grand Island, NY). Cells were maintained at of $37^{\circ} \mathrm{C}$ in $5 \% \mathrm{CO}_{2}$.

Materials. EGF, BTC, and Neuregulin 4 (NRG4) were purchased from Prospec-Tany TechnoGene Ltd (Rehovot, Israel). MM-121 (seribantumab) was provided by Merrimack Pharmaceuticals, Inc, (Cambridge, MA) (Schoeberl et al., 2009). All other chemicals were purchased from Sigma-Aldrich (St. Louis, MO), unless otherwise noted.

Single Cell Growth Assay. hTCEpi cells were plated at a density of 30 cells/well in a 12-well tissue culture dish in low-serum media (25\% growth media and $75 \%$ serum-free media). Cells were grown to the eight-cell stage then treated with EGF or BTC (1.6 nM) or no ligand. Photographs of the cell colonies were taken every 24 hours. At each time point, the number of cells per colony were counted and the area the colony covered was measured using ImageJ software (Schneider et al., 2012).

Competition Binding. All radioligand binding experiments used binding buffer [Dulbecco's modified Eagle's medium (without bicarbonate), $10 \mathrm{mM}$ NaHEPES, 0.1\% BSA, pH 7.4]. Confluent 12-well dishes of hTCEpi cells were incubated with varying concentrations of cold ligand (EGF, BTC, and NRG4) and ${ }^{125}$ I-EGF (specific activity, $150-200 \mu \mathrm{Ci} / \mu \mathrm{g} ; \sim 10,000 \mathrm{cpm} / 10 \mu \mathrm{l}$; cat. no. NEX160; PerkinElmer Life and Analytical Sciences, Boston, MA). Cells were incubated on ice for 2 hours, allowing a steady-state binding while preventing membrane trafficking. Cells were washed four times with radioligandbinding buffer and solubilized in $0.1 \%$ SDS $/ 0.1 \mathrm{~N} \mathrm{NaOH}$, and the radioactivity level of each sample was determined using a Beckman Coulter (Brea, CA) gamma counter. Data are plotted as the percentage of maximal radioactivity, as determined from cells with no competitive ligand added. $\mathrm{IC}_{50}$ values were calculated with a one-site model using GraphPad Prism version 6.05 (GraphPad Software, La Jolla, CA).

pH Dissociation Binding. Confluent 12-well dishes of hTCEpi cells were incubated with ${ }^{125} \mathrm{I}-\mathrm{EGF}$ (specific activity, $150-200 \mu \mathrm{Ci} / \mu \mathrm{g}$; cat. no. NEX160; PerkinElmer Life and Analytical Sciences) or ${ }^{125} \mathrm{I}-\mathrm{BTC}$ (custom synthesis; specific activity $94 \mu \mathrm{Ci} / \mu \mathrm{g} ; \sim 10,000$ $\mathrm{cpm} / 10 \mu \mathrm{l}$; PerkinElmer Life and Analytical Sciences). Cells were incubated on ice for 2 hours, allowing a steady-state distribution of the ligands. The $\mathrm{pH}$ of radioligand-binding buffer was adjusted with $\mathrm{NaOH}$ or $\mathrm{HCl}$ to bring the buffer to the desired $\mathrm{pH}$ (2-8). $\mathrm{pH}$-adjusted buffer was used to wash the cells to remove excess ligand and dissociate the ligand from the receptor. Cells were solubilized in $0.1 \% \mathrm{SDS} / 0.1 \mathrm{~N} \mathrm{NaOH}$, and the associated radioactivity in each sample was determined using a Beckman Coulter gamma counter. Data are plotted as the average ( \pm S.D.) percentage of radioactivity relative to maximal binding.

Western Blot with Phospho-Specific Antibodies. hTCEpi cells were washed twice with PBS pH 7.4 and incubated with serumfree Keratinocyte serum-free media for 2 hours. Cells were treated with the indicated ligand concentration at the indicated time. Treated cells were subjected to two quick washes with PBS pH 7.4 and equilibrated to $4^{\circ} \mathrm{C}$ on ice. Cells were harvested in lysis buffer [50 mM NaCl, 1\% NP40, 0.5\% deoxycholate, 0.1\% SDS, $50 \mathrm{mM}$ Tris (pH 8.0), $10 \mathrm{mM}$ sodium pyrophosphate, $100 \mathrm{mM}$ sodium fluoride] supplemented with $2 \mathrm{mM}$ phenylmethylsulfonyl fluoride, and solubilized with end-over-end rotation for 10 minutes at $4^{\circ} \mathrm{C}$. Insoluble material was removed by centrifugation for 10 minutes at $4^{\circ} \mathrm{C}$ and maximum speed (21,130 relative centrifugal force) in a microcentrifuge (model 5424R; Eppendorf, Hamburg, Germany). Equivalent amounts of cell lysate were resolved by SDS-PAGE and transferred to nitrocellulose. The indicated proteins were immunoblotted using the following antibodies: EGFR (SC-03) and EGFR (A-10) antibodies obtained from Santa Cruz Biotechnology (Dallas, TX), site-specific phospho-EGFR antibodies (pY998, pY1045, pY1068, and pY1148) were from Cell Signaling Technology (Danvers, MA), and $\alpha$-tubulin antibodies were from Sigma-Aldrich. After incubation with the appropriate horseradish peroxidase-conjugated secondary antibody, 
immunoreactive proteins were visualized with enhanced chemiluminescence and a Fotodyne (Hartland, WI) imaging system .

Immunoprecipitations. Cell lysates (500 $\mu \mathrm{g}$; see Western Blot with Phospho-Specific Antibodies section) were incubated with either a pool of phosphotyrosine antibodies (pY20, sc-508; pY99, sc-7020; from Santa Cruz Biotechnology; 4G10, 05-321; EMD Millipore, Billerica, MA) or ErbB3 antibodies [sc-285, sc-7390, sc-415; Santa Cruz Biotechnology; HER3/ErbB3 (D22C5) XP; Cell Signaling Technology] while rotated end over end at $4^{\circ} \mathrm{C}$ overnight. Protein A/G agarose beads (Santa Cruz Biotechnology) washed with lysis buffer were added and rotated end over end at $4^{\circ} \mathrm{C}$ for 2 hours. Lysates were centrifuged, and a sample of the supernatant was kept as a pass-through, the rest discarded. Then, lysis buffer was used to wash the beads three times. To elute the protein from the beads, SDS sample buffer was added to the samples and heated to $100^{\circ} \mathrm{C}$ for 3 minutes. The immunoprecipitates were divided into thirds, separated by SDS-PAGE, transferred to nitrocellulose, and detected with the indicated antibody: EGFR (Santa Cruz Biotechnology); ErbB2 and ErbB3 (Cell Signaling Technology); ErbB3 (Santa Cruz Biotechnology/Cell Signaling Technology); or PY99/phosphotyrosine (Santa Cruz Biotechnology), as described previously.

PLA. hTCEpi cells were plated on $\mathrm{NaOH}$ cleaned 12-mm-round no. 1 coverslips in a 24 -well plate and grown to $70 \%-80 \%$ confluency. Serum-starved cells were treated with media, EGF (16 nM), or BTC $(16 \mathrm{nM})$ at $37^{\circ} \mathrm{C}$ for 15 minutes. Coverslips were washed with $\mathrm{PBS}^{++}$

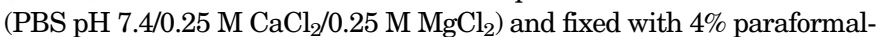
dehyde/PBS ${ }^{++}$for 5 minutes on ice and 15 minutes at room temperature. Cells were permeabilized with $0.1 \%$ saponin $/ 0.5 \%$ fetal bovine serum $/ \mathrm{PBS}^{++}$for 15 minutes, and washed three times in $\mathrm{PBS}^{++}$. EGFRErbB3 heterodimers were detected using a PLA kit (DuoLink; SigmaAldrich) according to manufacturer directions. Primary antibodies EGFR (E114, RabMAb; Abcam, Cambridge, UK) and ErbB3 (2F12; Thermo Fisher Scientific, Waltham, MA) were used independently and jointly, as indicated in experiments. Fluorescence images and quantification were acquired using a Nikon Eclipse Ti-E widefield microscope and Nikon NIS Elements software (Nikon, Melville, NY).

Transwell Migration Assay. The 100,000 hTCEpi cells in serum-free media (Keratinocyte Defined Media without growth supplement, $100 \mu \mathrm{l}$; Invitrogen) with or without $170 \mu \mathrm{g} / \mathrm{ml}$ MM-121 were plated in the upper chamber of an $8-\mu \mathrm{m}$ polycarbonate membrane, 6.5-mm insert (Corning, Inc., Corning, NY) for 2 hours at $37^{\circ} \mathrm{C}$. After incubation, the lower chamber contained $600 \mu \mathrm{l}$ of serum-free media containing the indicated concentrations of growth factor with or without MM-121 [seribantumab (a gift of Merrimack Pharmaceuticals)]. Cells were allowed to migrate for 16 hours at $37^{\circ} \mathrm{C}$ in $5 \% \mathrm{CO} 2$. Migrated cells were determined by fixing the cells in methanol, staining in Giemsa, and counting the migrated cells under a microscope (model TE-2000; Nikon, Tokyo, Japan) with a $60 \times$ objective (Vedham et al., 2005).

siRNA. The siRNAs were obtained from the following sources: scramble control siRNA, EGFR, and ErbB2 were acquired from IDTDNA (Coralville, IA); and ErbB3 Silencer Select was acquired from Thermo Fisher Scientific). hTCEpi cells were transfected with the indicated siRNA by Amaxa electroporation, as described previously (Rush and Ceresa, 2013). Cells were allowed to recover for 48 hours. Cells were assayed by Western blot for knockdown, phosphorylation of receptors, or cell migration, as described in Materials and Methods.

Statistical Analyses. Statistical tests are indicated in the figure legends and were performed using GraphPad Prism version 5.0.

\section{Results}

BTC Induces More Cell Migration than EGF. Our previous studies identified BTC-mediated in vitro corneal epithelial wound healing as significantly more robust than EGF-mediated in vitro corneal epithelial wound healing
(Peterson et al., 2014). Corneal epithelial wound healing is a multifaceted process that includes cell migration and proliferation (Stepp et al., 2014). The first goal for understanding the molecular basis for the enhanced activity of BTC was to determine whether the ligand was augmenting cell migration, cell proliferation, or both processes. To assess the ligand effect on proliferation and migration, hTCEpi cells were plated as single cells and cultured in media alone or in media supplemented with EGF or BTC. The same colony was photographed over 72 hours (Fig. 1A). From these images, the number of cells and their total area were determined (Fig. 1, B and C). Despite no increase in cell number, BTC-treated cultures covered a larger area of the dish compared with those grown in EGF or media alone, indicating the BTC-treated cells were more migratory.

To supplement that assay, a transwell assay was used to directly measure cell migration in response to the growth factors (Fig. 1D). BTC had a 6 -fold increase in cell migration compared with a 4-fold increase with EGF. Together, these assays demonstrate that BTC stimulates cell migration more than EGF.

BTC Binds EGFR with Lower Affinity. BTC is universally accepted to bind both EGFR and ErbB4 (Beerli and Hynes, 1996; Riese et al., 1996), but others report (Alimandi et al., 1997; Oh et al., 2011) that BTC can bind/activate ErbB2 and ErbB3 as well. hTCEpi cells do not express ErbB4 (Peterson et al., 2014), so we restricted our focus to the EGFR. To determine whether EGF and BTC were binding the same receptor, we performed an ${ }^{125}$ I-EGF competition binding assay. EGF, BTC, and NRG4 as a negative control, were used to compete for radiolabeled $\mathrm{EGF}$ (Fig. $2 \mathrm{~A}) . \mathrm{EGF}\left(\mathrm{IC}_{50}=\sim 2 \mathrm{nM}\right)$ was able to compete for ${ }^{125}$ I-EGF binding with a 30 -fold higher affinity than BTC $\left(\mathrm{IC}_{50}=\sim 60 \mathrm{nM}\right) ; 100 \mathrm{nM}$ NRG4 was unable to displace ${ }^{125}$ I-EGF binding, which is consistent with its role as an ErbB4-specific ligand (Fig. 2A). These data indicate that EGF and BTC compete for the same binding site and that BTC has a lower affinity for the EGFR.

Next, we examined whether EGFR binding of BTC and EGF had differing sensitivities to $\mathrm{pH}$. As the ligand-receptor complex progresses through the endocytic pathway, it moves through an increasingly acidic environment. Ligand binding is one of the factors that determines whether the receptor recycles back to the plasma membrane or is targeted to the lysosome. Approximately $50 \%$ of bound ${ }^{125}$ I-EGF dissociated at $\mathrm{pH} 5.8$, whereas a $\mathrm{pH}$ of 4.3 was required to dissociate $50 \%$ of the bound ${ }^{125} \mathrm{I}$-BTC (Fig. 2B). These values led to the prediction that $\sim 50 \%$ of EGF-EGFR complexes will dissociate in the early endosome ( $\mathrm{pH} \sim 5.9-6.8$ ) (Yamashiro and Maxfield, 1987), whereas the BTC-EGFR complex is not predicted to have appreciable dissociation, even in the lysosome ( $\mathrm{pH} \sim 4.5-5.0$ ) (Yamashiro and Maxfield, 1987).

BTC Induces Less EGFR Phosphorylation but More ErbB3 Phosphorylation. Consistent with the reduced affinity, BTC was a weaker activator of EGFR, compared with EGF. Using ligand concentrations that produced maximal cell migration (Peterson et al., 2014), a time course of tyrosine phosphorylation was used as a readout of EGFR activity (Fig. 3). hTCEpi cells treated with BTC compared with those treated with EGF had reduced EGFR phosphorylation at all four of the tyrosine residues examined (Fig. 3A). Similar trends were observed in MDA-MB-468 cells, a metastatic breast cancer cell line with comparable levels of EGFR (Fig. 3B). It should be noted that supraphysiological 

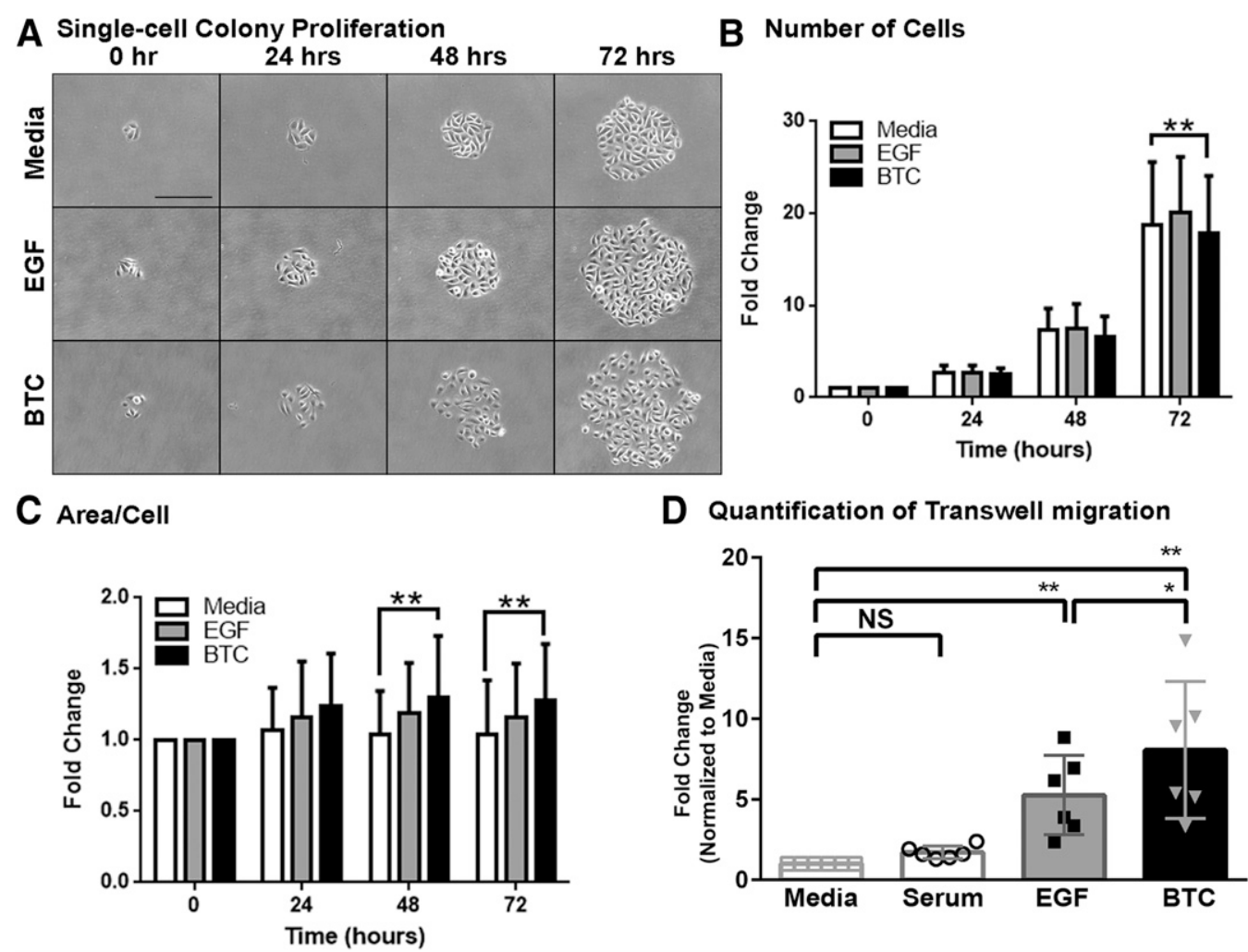

Fig. 1. BTC is a more efficacious activator of cell migration than EGF. (A-C) hTCEpi cells were plated as single cells ( 30 cells/35 mm dish) in media alone, $1.6 \mathrm{nM} \mathrm{EGF}$, or $1.6 \mathrm{nM}$ BTC. (A) Once cell colonies formed, they were imaged at 24-hour intervals for 72 hours. Shown are representative data from an experiment repeated three times ( $\sim 25-70$ colonies for each condition). Scale bar, $200 \mu \mathrm{m}$. (B) Data from three separate experiments were plotted as the average \pm S.D. fold change as the number of cells per colony. (C) Data from three separate experiments were plotted as the average \pm S.D. fold change in area per cell. (D) hTCEpi cell migration was measured using a transwell assay after migrating for 16 hours, as described in Experimental Procedures. Cells were incubated in serum-free media with no additions (Media), serum (Serum), $1.6 \mathrm{nM}$ EGF, or 1.6 nM BTC. Plotted are the fold changes (average \pm S.D.) in cell migration (relative to serum-free media) for each condition $(n=6)$. Data were analyzed using a two-way ANOVA with a Tukey's post hoc analysis. $* P<0.05 ; * * P<0.01$; NS, not significant.

concentrations of BTC and EGF produce comparable levels of EGFR phosphorylation (Peterson et al., 2014). The differences in EGFR phosphorylation were revealed using ligand concentrations $(1.6 \mathrm{nM})$ that produce the greatest biologic response (Peterson et al., 2014), more closely reflect physiologic concentrations (Peterson et al., 2014), and are closer to the $\mathrm{K}_{\mathrm{d}}$ (dissociation constant) values for the EGFR ligands (Schlessinger, 1986; Watanabe et al., 1994).

The decrease in EGFR phosphorylation led us to an alternative explanation. We hypothesized that EGF promoted EGFR homodimers, whereas BTC preferentially formed EGFR-ErbB3 heterodimers. The model accounts for the observations that: 1 ) the ligands can compete for binding with one another (Jones et al., 1999; Macdonald-Obermann and Pike, 2014), 2) the EGFR-ErbB3 heterodimers would have reduced EGFR phosphorylation due to ErbB3 being kinase impaired (Guy et al., 1994), and 3) ErbB3 activation is associated with induced proliferation and migration (Lyons et al., 2005; Reschke et al., 2008; Sathyamurthy et al., 2015).

To test whether BTC could differentially signal through other ErbB receptors, we examined receptor tyrosine phosphorylation (Fig. 4, A-C). hTCEpi cells were treated with media alone or media supplemented with EGF or BTC. For these biochemical experiments, higher concentrations of ligand were used to drive receptor dimerization and enhance the signal-to-noise ratio. Denatured cell lysates were immunoprecipitated with an anti-phosphotyrosine antibody and probed for the presence of the three endogenous ErbB receptors (Fig. 4A). BTC treatment led to decreased phosphorylation of EGFR and ErbB2 when compared with EGF, whereas only BTC treatment resulted in significant ErbB3 phosphorylation (Fig. 4D). Significant ErbB3 phosphorylation by BTC was also observed when cell lysates were immunoprecipitated using an ErbB3 antibody and immunoblotted with an anti-phosphotyrosine antibody (Fig. 4, B and E).

hTCEpi cells were transduced using ErbB2-specific short hairpin RNA (shRNA) to examine the possibility of ErbB2 involvement in ErbB3 phosphorylation. BTC-mediated activation of ErbB3 is unaffected by the loss of ErbB2 (calculated to be 86\% knockdown), further indicating that ErbB2 is dispensable in BTC activation of ErbB3 (Fig. 4, C and F). To determine whether these observations were specific to hTCEpi cells, we treated MDA-MB-468 cells with EGF and BTC, and then immunoprecipitated phosphorylated tyrosines and immunoblotted for the same three ErbB receptors available in hTCEpi cells (Supplemental Fig. 1, A-D). MDA-MB-468 cells express EGFR and ErbB3, but do not express detectable levels of ErbB2 or ErbB4 (Khan et al., 2010). As with the hTCEpi cells, BTC treatment resulted in less EGFR tyrosine phosphorylation and induced ErbB3 tyrosine phosphorylation.

BTC Promotes ErbB3 Phosphorylation and EGFRErbB Heterodimers. These biochemical data provide evidence that BTC causes ErbB3 phosphorylation but do not demonstrate a direct interaction. Another possibility is that 
A 125 I-EGF Competition binding

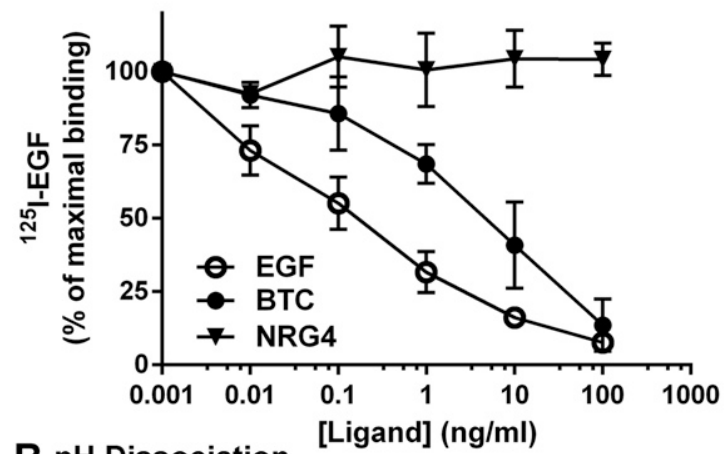

B pH Dissociation

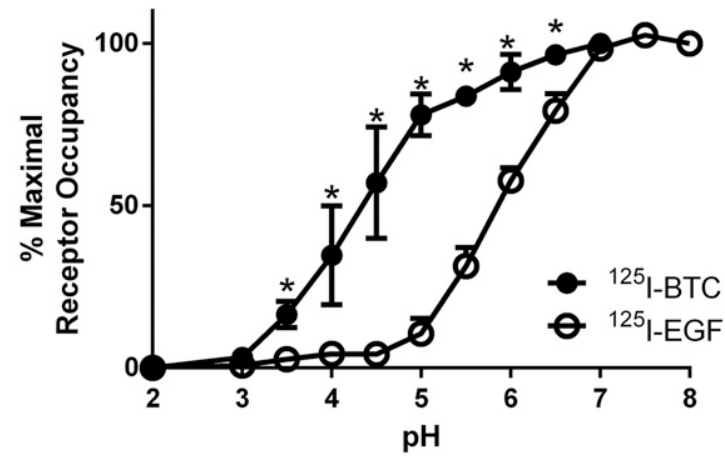

Fig. 2. BTC and EGF bind EGFRs with different affinities and $\mathrm{pH}$ sensitivities. (A) ${ }^{125}$ I-EGF competition binding using varying concentrations of EGF, BTC, or NRG4 as indicated. (B) pH sensitivity of ${ }^{125}$ I-EGF and ${ }^{125} \mathrm{I}$-BTC binding. Cells were incubated with radioligand at $\mathrm{pH} 7.4$ and $4{ }^{\circ} \mathrm{C}$ until steady-state binding was achieved. Cells were then incubated in binding buffer at varying $\mathrm{pH}$ values at $4^{\circ} \mathrm{C}$ for 30 minutes, then were washed three times in ice-cold buffer of the same $\mathrm{pH}$. Cells were solubilized and associated radioactivity was measured. For (A and B), data are plotted as the percentage of maximal radioligand binding (average \pm S.D., $n=3$ ). $* P<0.01$.

ErbB3 phosphorylation is mediated by a nonreceptor kinase that is activated by the liganded EGFR. To determine whether BTC caused an increase in EGFR-ErbB3 heterodimers, we performed PLA (Fig. 5). PLA is an antibody-based detection/amplification system that will reveal proteinprotein interactions when they are within $40 \mathrm{~nm}$. hTCEpi cells were treated with media alone, or media supplemented with EGF or BTC, fixed and subjected to the PLA protocol (see Experimental Procedures). When cells were screened for the presence of EGFR-ErbB3 heterodimers, EGF produced a 1.1fold increase in heterodimers, and BTC produced a 2.6-fold increase. Together, the receptor phosphorylation data and the PLA support the notion that EGF-treated cells have fewer EGFR-ErbB3 heterodimer interactions when compared with those cells treated with BTC.

Inhibition of ErbB3 Preferentially Attenuates BTCMediated Cell Migration. Finally, we wanted to determine whether the EGFR-ErbB3 heterodimers were responsible for the increase in cell mobility that was seen with BTC treatment. We monitored ligand-dependent cell migration using a transwell migration assay in the absence and presence of the ErbB3 antagonist antibody MM-121 (seribantumab) (Schoeberl et al., 2009) (Fig. 6). In the presence of MM-121, BTC-treated cells no longer migrated more than EGF-treated cells.

To determine the contribution of ErbB family members to BTC-mediated migration, we knocked down EGFR, ErbB2, and ErbB3 (Fig. 6B) individually. Transfection of hTCEpi cells with these siRNAs attenuated the expression of EGFR, ErbB2, and ErbB3 by $49 \%, 78 \%$, and $91 \%$, respectively. The decreased levels of ErbB3 eliminated the significant BTC-mediated migration advantage when compared with EGF (Fig. 6C). The loss of any of the three ErbB RTKs decreases the advantage of BTC treatment over EGF (Fig. 6C). These data are consistent with all ErbB family members contributing to BTC-mediated signaling.

\section{Discussion}

In this article, we demonstrate that EGFR-related growth factors differ in their ability to induce phosphorylation, direct specific receptor dimerization, and effect cell physiology. Multiple laboratories have reported that BTC treatment can generate a greater response than EGF (Beerli and Hynes, 1996; Schoeberl et al., 2009; Knudsen et al., 2014;

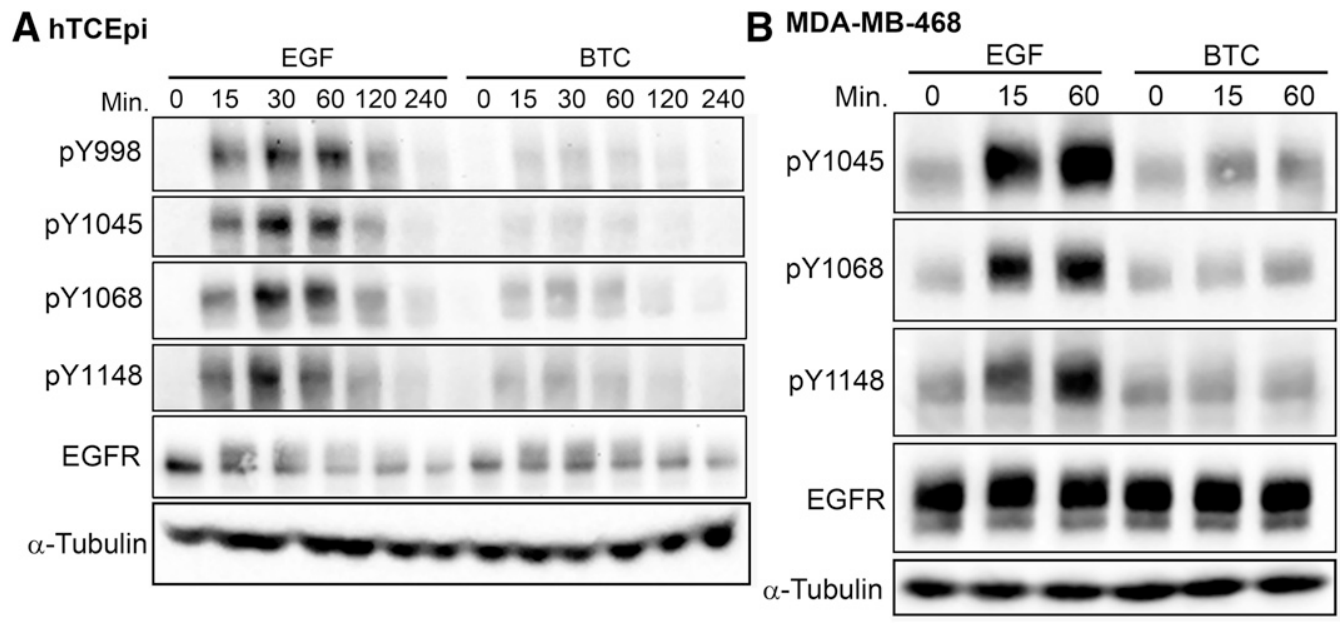

Fig. 3. BTC induces less EGFR phosphorylation than EGF in hTCEpi and MDA-MB-468 cells. (A and B) Cells were treated with $1.6 \mathrm{nM}$ EGF or $1.6 \mathrm{nM}$ BTC for the indicated times. Cell lysates were prepared and immunoblotted with the indicated EGFR phosphotyrosine-specific antibodies (pY998, pY1045, pY1068, pY1148), total EGFR, or $\alpha$-tubulin. (A) hTCEpi cell representative blots from three experiments. (B) MDA-MB-468 cell representative blots from three experiments. 


\section{A \\ hTCEpi \\ B hTCEpi}

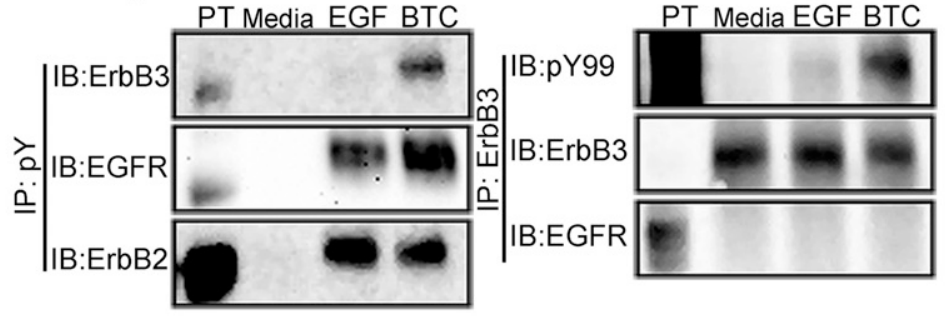

C hTCEpi:ErbB2 Knockdown

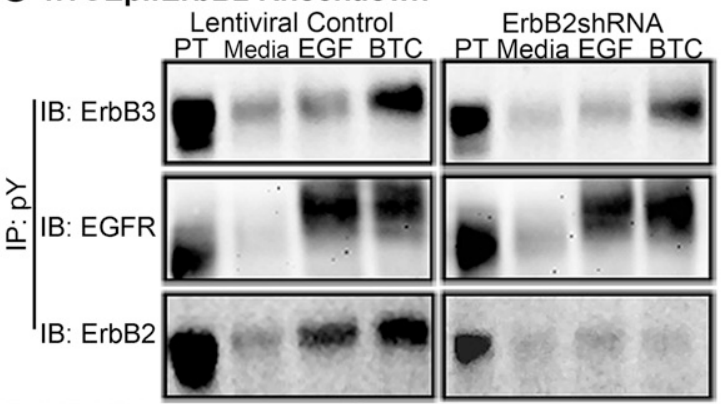

D hTCEpi: pY:IP (Quant.)

E hTCEpi: ErbB3:IP (Quant.)

F hTCEpi:ErbB2 Knockdown (Quantification)
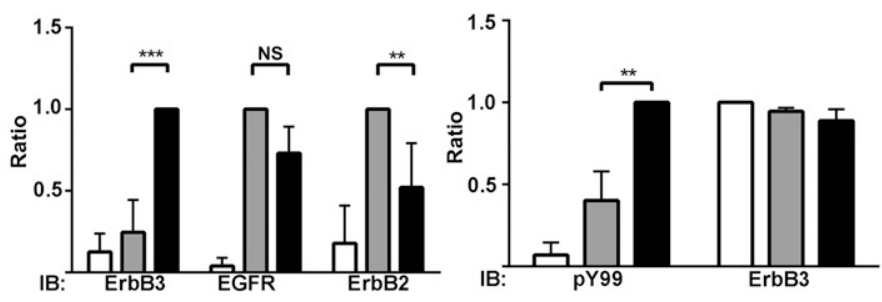

$\square$ Media $\square$ EGF

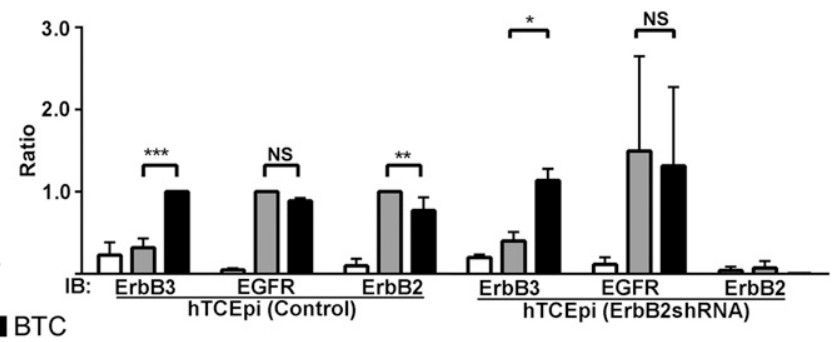

Fig. 4. BTC induces ErbB3 phosphorylation in hTCEpi cells without ErbB2 interaction. (A-C) hTCEpi were treated with serum-free media alone, $16 \mathrm{nM}$ EGF, or $16 \mathrm{nM}$ BTC for 15 minutes, as indicated. Cell lysates were prepared under denaturing conditions, and the indicated proteins were immunoprecipitated using anti-phosphotyrosine (A) or anti-ErbB3 (B) antibodies. The pass-through (PT) and immunoprecipitates were resolved by 7.5\% SDS-PAGE and immunoblotted with the indicated antibodies. Shown are representative blots from an experiment performed at least three times. (C) Transduced ErbB2-shRNA and shRNA control (Materials and Methods) hTCEpi cells were treated the same as (A and B). Shown are representative blots from three replicates. (D-F) Immunoblots from (A-C) were quantified using ImageJ (National Institutes of Health). Data are presented as the average ratio $( \pm$ S.D. $)$ of each treatment to the most abundant protein. Blots were analyzed with a two-way ANOVA, Holm-Sidak multiple-comparisons test. $* P<0.05 ; * * P<0.01 ; * * * P<0.001 \mathrm{IB}$, immunoblot; IP, immunoprecipitation; NS, not significant.

Peterson et al., 2014). We show that, in line with those findings, BTC enhances cell migration more than EGF (Figs. 1 and 6), which is a fundamental component of corneal wound healing (Figs. 1 and 6). Furthermore, this effect is not due to enhanced BTC binding to or phosphorylation of the EGFR (Figs. 2-4), because these responses are reduced with BTC treatment.

Rather, BTC binding to the EGFR biases the receptor to heterodimerize with ErbB3, resulting in ErbB3 phosphorylation (Figs. 3-5; Supplemental Fig. 1). Importantly, BTC-mediated ErbB3 phosphorylation is independent of ErbB2. These heterodimers contribute to cell migration, because ErbB3 antagonists and knockdown inhibit BTC-mediated migration to a greater extent than EGF-mediated migration (Fig. 6).
We propose a model in which EGF and BTC both bind the EGFR, but differentially bias the dimerization partner of the ligand-bound receptor. This ligand-biased signaling is well established among G-protein-coupled receptors (Kinzer-Ursem and Linderman, 2007; Zidar et al., 2009; Luttrell and Kenakin, 2011). In the cell, monomeric ErbB RTKs and their ligands are in a biochemical equilibrium. The binding of ligand shifts the equilibrium of the receptors from a monomeric to a dimeric form. Our data here support a model in which the ligand-EGFR complex directs whether the dimers are homodimers or heterodimers. There is an additional layer of complexity to this model when applying it to other cell lines, which express the ErbB RTKs at different levels.

\section{A PLA: EGFR:ErbB3 Heterodimers}

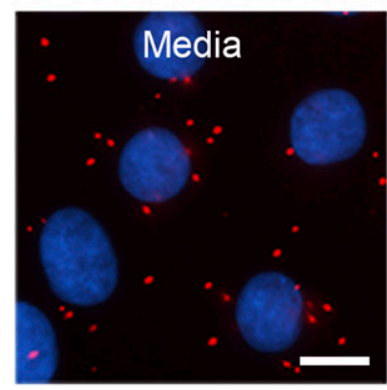

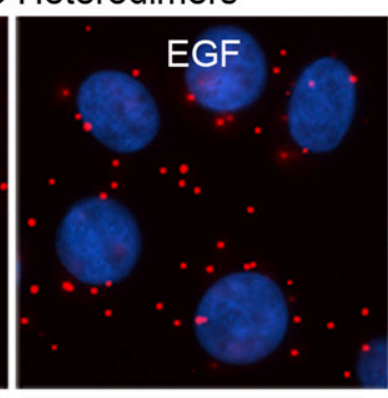

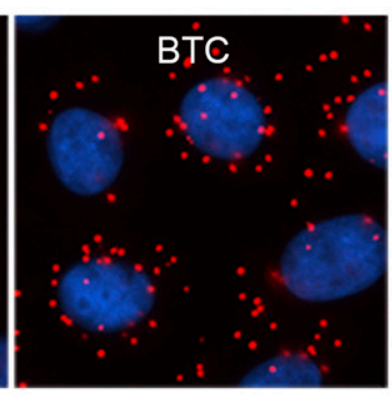

B PLA: EGFR:ErbB3 (Total)

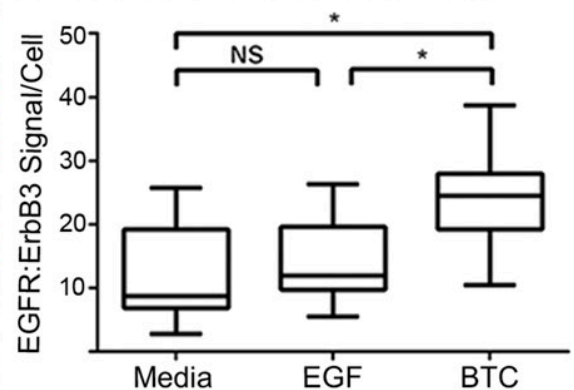

Fig. 5. BTC induces EGFR-ErbB3 heterodimers in hTCEpi cells. EGFR-EGFR or EGFR-ErbB3 dimerization was visualized by PLA. After serum starving cells for 2 hours, hTCEpi cells were treated with serum-free media alone or with $16 \mathrm{nM} \mathrm{EGF} \mathrm{or} \mathrm{BTC} \mathrm{for} 15$ minutes at $37^{\circ} \mathrm{C}$. After treatment, cells were fixed and probed using the PLA as described in Materials and Methods. (A) Representative micrographs of cells probed for EGFR-ErbB3 heterodimers. (B) Data from three independent experiments were quantified (50-100 cells/experiment) and are presented as a box-and-whisker plot of the total number of signals per cell and analyzed with a one-way ANOVA, Holm-Sidak multiple-comparisons test. $* P<0.05$; NS, not significant. 
A ErbB3 inhibitor migration

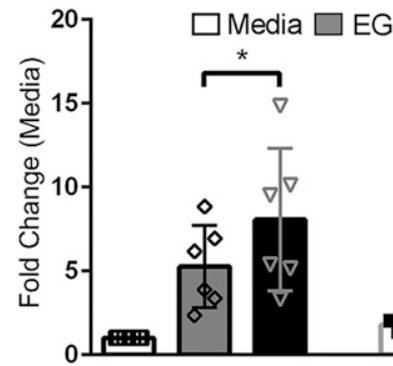

(-) MM-121

C ErbB knockdown migration

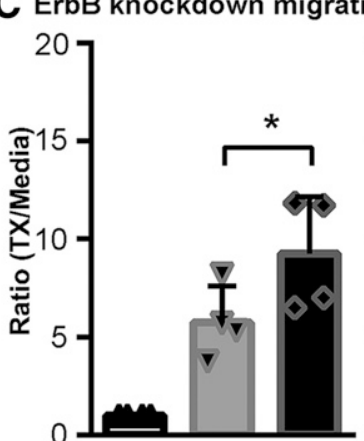

siRNA siCON

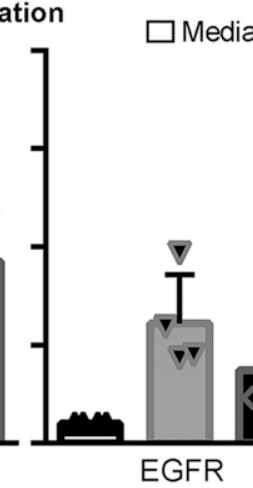

(+) MM-121

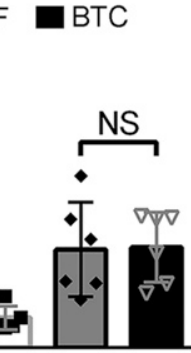

B ErbB knockdown migration

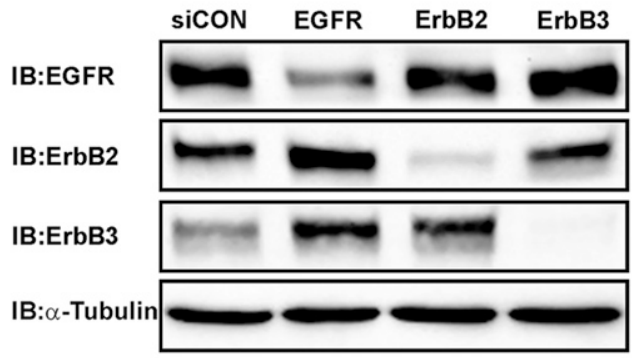

BTC

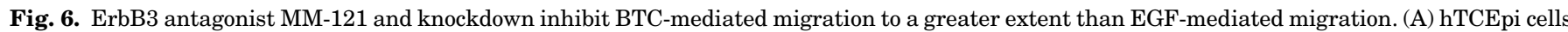

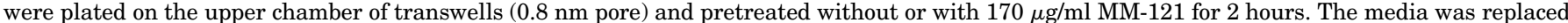

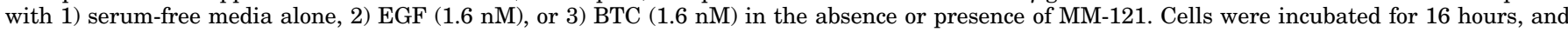

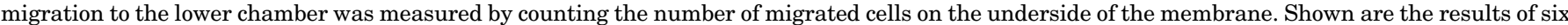

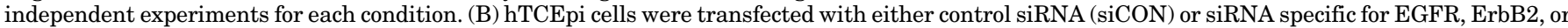

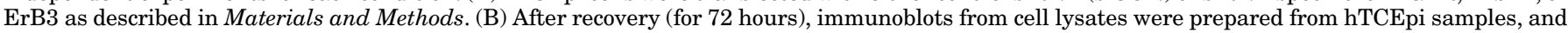

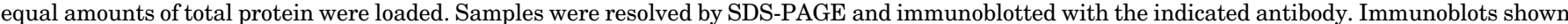

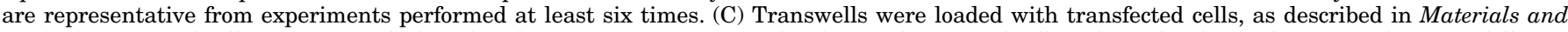

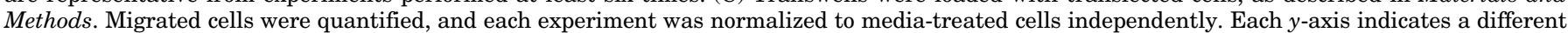

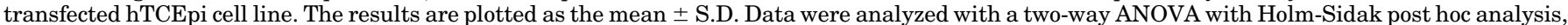
$* P<0.05$. NS, not significant.

Reports in the literature have been inconsistent regarding how BTC induces ErbB3 phosphorylation (Riese et al., 1995; Pinkas-Kramarski et al., 1996; Riese et al., 1996; Chiba, 2004; Steinkamp et al., 2014). We postulate that the absolute and relative amounts of EGFR and ErbB3 in different cell lines are a potential source for these discrepancies. Since BTC drives the formation of EGFR-ErbB3 heterodimers, if EGFRs are in vast excess, once the maximum number of heterodimers are formed, the remaining liganded EGFRs are able to form homodimers or ErbB2 heterodimers. These dimers may mask the contribution of EGFR-ErbB3 heterodimer. Our data support this hypothesis, because the loss of EGFR and ErbB2 also decreased the significant advantage that BTC treatment had on migration (Fig. 6). hTCEpi cells have sufficient levels of EGFR and ErbB3 to produce functional EGFR-ErbB3 heterodimers, as is evident by the decreased cell migration in response to ErbB3 antagonist and ErbB3 knockdown. Cells that do not express ErbB3 may not show differences in responses to EGF and BTC.

Several previous studies support this model. First, Liu et al. (2012) report that a single ligand is sufficient to activate EGFR dimers. Although this is well accepted for ErbB RTKs that heterodimerize with the ligandless ErbB2, it is important to note that it can extend to the other family members that undergo conformational changes to dimerize. Second, our findings that receptors preferentially form dimer partners are supported by the work of Macdonald-Obermann et al. (2013) and Macdonald-Obermann and Pike (2014). Our endogenous receptor model proposes that these preferences can be shifted by the activating ligand. As indicated from our PLA experiments, BTC preferentially drives the formation of the EGFR-ErbB3 complex.

It was fortunate that hTCEpi and MDA-MB-468 cells do not express ErbB4, another target for BTC binding. With the addition of ErbB4, the model would have to take into consideration the binding affinities for EGFR versus ErbB4. Although ErbB2 does not bind BTC, its contribution cannot be overlooked. It is likely that ErbB2 is also competing for binding to the liganded EGFRs. ErbB3 phosphorylation was unaffected by the loss of ErbB2, further supporting EGFR as the sole ligand-binding receptor in our system. The incomplete inhibition of ErbB3-initiated migration in response to MM-121 may be due to compensatory ErbB2 effects. The knockdown of each ErbB RTK had an increase in protein levels of the other ErbB RTKs when compared with control lysates, especially with EGFR knockdown (Fig. 6B). Other groups show that compensatory protein effects after the knockdown of similar proteins are possible and support this phenomenon (Krumins and Gilman, 2006; Rossi et al., 2015). This effect was also supported in the receptor knockout cell lines where the loss of EGFR, ErbB2, and ErbB3 decreased the migration advantage that BTC has over EGF. Although ErbB2 is not 
necessary to activate ErbB3 with BTC, it contributes to BTCmediated migration, similar to ErbB3 (Fig. 6C). More rigorous mathematical modeling and experimentation are required to account for the complexities of the entire ErbB RTK family.

Although it has been previously appreciated that ligands can direct dimer formation, it was largely attributed to differences in which receptor the ligand bound (MacdonaldObermann and Pike, 2014); our model proposes that two different ligands are binding the same receptor, but forming different heterodimer populations. How would ligand binding direct the dimerization partner? One possibility is that human EGF and BTC have limited sequence similarity $(\sim 36 \%)$ as well as low predicted three-dimensional structural homology as measured by solvent accessibility surfaces (López-Torrejón et al., 2002). Alternatively, the size of the ligand may affect receptor conformation. The processed form of BTC is $9 \mathrm{kDa}$, and EGF is $6.6 \mathrm{kDa}$ (Harris et al., 2003). A stretch of $\sim 50$ amino acids directly binds to the ligand-binding domain of the receptor (Seno et al., 1996). The size and/or charge of the remaining 30 amino acids may sterically hinder BTC-EGFR homodimerization. Fundamental differences in binding are supported by our observed differences in radioligand competition binding and $\mathrm{pH}$ sensitivity for the two ligands (Fig. 2).

Conclusion. The physiologic significance of individual ErbB RTK ligands is likely important, as indicated by knockout animals. Using cell culture models, we were able to dissect how EGF and BTC can form different ErbB receptor combinations and elicit specific cell biologic responses. We propose a new model for how BTC binding to the EGFR biases it in the formation of EGFR-ErbB3 heterodimers. These heterodimers are distinct from EGFR homodimers in their ability to enhance cell migration despite less EGFR phosphorylation. This model may have important implications in the prognosis of cancer. Many cancers are characterized by the overexpression of individual ErbB family members and specific ErbB ligands (Hynes and MacDonald, 2009). Based on the evidence that ligand binding affects receptor dimerization and the resulting cell biology, assessing receptor levels and local levels of growth factor may predict the invasiveness of the cancer.

\section{Authorship Contributions}

Participated in research design: Rush, Peterson, Ceresa.

Conducted experiments: Rush, Peterson.

Contributed new reagents or analytical tools: Rush, Peterson.

Performed data analysis: Rush, Peterson, Ceresa.

Wrote or contributed to the writing of the manuscript: Rush, Peterson, Ceresa.

\section{References}

Alimandi M, Wang LM, Bottaro D, Lee CC, Kuo A, Frankel M, Fedi P, Tang C, Lippman M, and Pierce JH (1997) Epidermal growth factor and betacellulin mediate signal transduction through co-expressed ErbB2 and ErbB3 receptors. EMBO $J$ 16:5608-5617.

Beerli RR and Hynes NE (1996) Epidermal growth factor-related peptides activate distinct subsets of ErbB receptors and differ in their biological activities. J Biol Chem 271:6071-6076.

Ceresa BP, Gosney JA, Jackson NM, and Rush JS (2016) Epidermal growth factor receptor, in Encyclopedia of Signaling Molecules, pp 1-11, Springer New York, New York.

Chen JC, Zeng FH, Forrester SJ, Eguchi S, Zhang MZ, and Harris RC (2016) Expression and function of the epidermal growth factor receptor in physiology and disease. Physiol Rev 6:1025-1069.

Chiba T (2004) What are the real roles of different erbB proteins in Barrett's Esophagus. Digestion 70:93-94.

Dahlhoff M, Dames PM, Lechner A, Herbach N, van Bürck L, Wanke R, Wolf E, and Schneider MR (2009) Betacellulin overexpression in transgenic mice improves glucose tolerance and enhances insulin secretion by isolated islets in vitro. Mol Cell Endocrinol 299:188-193.
Dahlhoff M, Schäfer M, Wolf E, and Schneider MR (2013) Genetic deletion of the EGFR ligand epigen does not affect mouse embryonic development and tissue homeostasis. Exp Cell Res 319:529-535.

Guy PM, Platko JV, Cantley LC, Cerione RA, and Carraway KL III (1994) Insect cellexpressed p180erbB3 possesses an impaired tyrosine kinase activity. Proc Natl Acad Sci USA 91:8132-8136.

Harris RC, Chung E, and Coffey RJ (2003) EGF receptor ligands. Exp Cell Res 284:2-13. Hynes NE and MacDonald G (2009) ErbB receptors and signaling pathways in cancer. Curr Opin Cell Biol 21:177-184.

Jackson LF, Qiu TH, Sunnarborg SW, Chang A, Zhang C, Patterson C, and Lee DC (2003) Defective valvulogenesis in HB-EGF and TACE-null mice is associated with aberrant BMP signaling. EMBO $J$ 22:2704-2716.

Jones JT, Akita RW, and Sliwkowski MX (1999) Binding specificities and affinities of egf domains for ErbB receptors. FEBS Lett 447:227-231.

Khan IH, Zhao J, Ghosh P, Ziman M, Sweeney C, Kung HJ, and Luciw PA (2010) Microbead arrays for the analysis of ErbB receptor tyrosine kinase activation and dimerization in breast cancer cells. Assay Drug Dev Technol 8:27-36.

Kinzer-Ursem TL and Linderman JJ (2007) Both ligand- and cell-specific parameters control ligand agonism in a kinetic model of $\mathrm{g}$ protein-coupled receptor signaling. PLOS Comput Biol 3:e6.

Knudsen SL, Mac AS, Henriksen L, van Deurs B, and Grøvdal LM (2014) EGFR signaling patterns are regulated by its different ligands. Growth Factors 32:155-163.

Krumins AM and Gilman AG (2006) Targeted knockdown of G protein subunits selectively prevents receptor-mediated modulation of effectors and reveals complex changes in non-targeted signaling proteins. J Biol Chem 281:10250-10262.

Li L, Seno M, Yamada H, and Kojima I (2001) Promotion of beta-cell regeneration by betacellulin in ninety percent-pancreatectomized rats. Endocrinology 142:5379-5385

Liu P, Cleveland TE 4th, Bouyain S, Byrne PO, Longo PA, and Leahy DJ (2012) A single ligand is sufficient to activate EGFR dimers. Proc Natl Acad Sci US A 109: 10861-10866.

López-Torrejón I, Querol E, Avilés FX, Seno M, de Llorens R, and Oliva B (2002) Human betacellulin structure modeled from other members of EGF family. $J \mathrm{Mol}$ Model 8:131-144.

Luetteke NC, Qiu TH, Fenton SE, Troyer KL, Riedel RF, Chang A, and Lee DC (1999) Targeted inactivation of the EGF and amphiregulin genes reveals distinct roles for EGF receptor ligands in mouse mammary gland development. Development 126:2739-2750.

Luttrell LM and Kenakin TP (2011) Refining efficacy: allosterism and bias in G protein-coupled receptor signaling, in Signal Transduction Protocols (Luttrell LM and Ferguson SSG eds) pp 3-35, Humana Press, Totowa, NJ.

Lyons DA, Pogoda HM, Voas MG, Woods IG, Diamond B, Nix R, Arana N, Jacobs J, and Talbot WS (2005) erbb3 and erbb2 are essential for schwann cell migration and myelination in zebrafish. Curr Biol 15:513-524.

Macdonald-Obermann JL, Adak S, Landgraf R, Piwnica-Worms D, and Pike LJ (2013) Dynamic analysis of the epidermal growth factor (EGF) receptor-ErbB2ErbB3 protein network by luciferase fragment complementation imaging. $J$ Biol Chem 288:30773-30784.

Macdonald-Obermann JL and Pike LJ (2014) Different epidermal growth factor (EGF) receptor ligands show distinct kinetics and biased or partial agonism for homodimer and heterodimer formation. $J$ Biol Chem 289:26178-26188.

Mann GB, Fowler KJ, Gabriel A, Nice EC, Williams RL, and Dunn AR (1993) Mice with a null mutation of the TGF alpha gene have abnormal skin architecture, wavy hair, and curly whiskers and often develop corneal inflammation. Cell 73:249-261.

Moon WS, Park HS, Yu KH, Park MY, Kim KR, Jang KY, Kim JS, and Cho BH (2006) Expression of betacellulin and epidermal growth factor receptor in hepatocellular carcinoma: implications for angiogenesis. Hum Pathol 37:1324-1332.

Oh YS, Shin S, Lee Y-J, Kim EH, and Jun H-S (2011) Betacellulin-induced beta cell proliferation and regeneration is mediated by activation of ErbB-1 and ErbB-2 receptors. PLoS One 6: 23894

Pathak BG, Gilbert DJ, Harrison CA, Luetteke NC, Chen X, Klagsbrun M, Plowman GD, Copeland NG, Jenkins NA, and Lee DC (1995) Mouse chromosomal location of three EGF receptor ligands: amphiregulin (Areg), betacellulin (Btc), and heparinbinding EGF (Hegfl). Genomics 28:116-118.

Peterson JL, Phelps ED, Doll MA, Schaal S, and Ceresa BP (2014) The role of endogenous epidermal growth factor receptor ligands in mediating corneal epithelia homeostasis. Invest Ophthalmol Vis Sci 55:2870-2880.

Pinkas-Kramarski R, Soussan L, Waterman H, Levkowitz G, Alroy I, Klapper L, Lavi S, Seger R, Ratzkin BJ, Sela M, et al. (1996) Diversification of Neu differentiation factor and epidermal growth factor signaling by combinatorial receptor interactions. EMBO J 15:2452-2467.

Reschke M, Mihic-Probst D, van der Horst EH, Knyazev P, Wild PJ, Hutterer M, Meyer S, Dummer R, Moch H, and Ullrich A (2008) HER3 is a determinant for poor prognosis in melanoma. Clin Cancer Res 14:5188-5197.

Riese DJ II, Bermingham Y, van Raaij TM, Buckley S, Plowman GD, and Stern DF (1996) Betacellulin activates the epidermal growth factor receptor and erbB-4, and induces cellular response patterns distinct from those stimulated by epidermal growth factor or neuregulin-beta. Oncogene 12:345-353.

Riese DJ II, van Raaij TM, Plowman GD, Andrews GC, and Stern DF (1995) The cellular response to neuregulins is governed by complex interactions of the erbB receptor family. Mol Cell Biol 15:5770-5776.

Robertson DM, Li L, Fisher S, Pearce VP, Shay JW, Wright WE, Cavanagh HD, and Jester JV (2005) Characterization of growth and differentiation in a telomerase-immortalized human corneal epithelial cell line. Invest Ophthalmol Vis Sci 46:470-478.

Rossi A, Kontarakis Z, Gerri C, Nolte H, Hölper S, Krüger M, and Stainier DYR (2015) Genetic compensation induced by deleterious mutations but not gene knockdowns. Nature 524:230-233.

Rush JS and Ceresa BP (2013) RAB7 and TSG101 are required for the constitutive recycling of unliganded EGFRs via distinct mechanisms. Mol Cell Endocrinol 381: 188-197. 
Sasada R, Ono Y, Taniyama Y, Shing Y, Folkman J, and Igarashi K (1993) Cloning and expression of cDNA encoding human betacellulin, a new member of the EGF family. Biochem Biophys Res Commun 190:1173-1179.

Sathyamurthy A, Yin DM, Barik A, Shen C, Bean JC, Figueiredo D, She JX, Xiong WC, and Mei L (2015) ERBB3-mediated regulation of Bergmann glia proliferation in cerebellar lamination. Development 142:522-532.

Schlessinger J (1986) Allosteric regulation of the epidermal growth factor receptor kinase. J Cell Biol 103:2067-2072.

Schneider CA, Rasband WS, and Eliceiri KW (2012) NIH Image to ImageJ: 25 years of image analysis. Nat Methods 9:671-675.

Schneider MR, Antsiferova M, Feldmeyer L, Dahlhoff M, Bugnon P, Hasse S, Paus R, Wolf E, and Werner S (2008a) Betacellulin regulates hair follicle development and hair cycle induction and enhances angiogenesis in wounded skin. J Invest Dermatol 128:1256-1265.

Schneider MR, Dahlhoff M, Herbach N, Renner-Mueller I, Dalke C, Puk O, Graw J, Wanke R, and Wolf E (2005) Betacellulin overexpression in transgenic mice causes disproportionate growth, pulmonary hemorrhage syndrome, and complex eye pathology. Endocrinology 146:5237-5246.

Schneider MR, Werner S, Paus R, and Wolf E (2008b) Beyond wavy hairs: the epidermal growth factor receptor and its ligands in skin biology and pathology. Am $J$ Pathol 173:14-24.

Schoeberl B, Pace EA, Fitzgerald JB, Harms BD, Xu L, Nie L, Linggi B, Kalra A, Paragas V, Bukhalid R, et al. (2009) Therapeutically targeting ErbB3: a key node in ligand-induced activation of the ErbB receptor-PI3K axis. Sci Signa 2:ra31.

Seno M, Tada H, Kosaka M, Sasada R, Igarashi K, Shing Y, Folkman J, Ueda M, and Yamada H (1996) Human betacellulin, a member of the EGF family dominantly expressed in pancreas and small intestine, is fully active in a monomeric form. Growth Factors 13:181-191.

Shing Y, Christofori G, Hanahan D, Ono Y, Sasada R, Igarashi K, and Folkman J (1993) Betacellulin: a mitogen from pancreatic beta cell tumors. Science 259: 1604-1607.

Singh B, Carpenter G, and Coffey RJ (2016) EGF receptor ligands: recent advances. F1000Res 5:F1000 Faculty Rev-2270.

Singh B and Coffey RJ (2014) Trafficking of epidermal growth factor receptor ligands in polarized epithelial cells. Annu Rev Physiol 76:275-300.

Srinivasan R, Benton E, McCormick F, Thomas H, and Gullick WJ (1999) Expression of the c-erbB-3/HER-3 and c-erbB-4/HER-4 growth factor receptors and their ligands, neuregulin-1 alpha, neuregulin-1 beta, and betacellulin, in normal endometrium and endometrial cancer. Clin Cancer Res 5:2877-2883.

Steinkamp MP, Low-Nam ST, Yang S, Lidke KA, Lidke DS, and Wilson BS (2014) erbB3 is an active tyrosine kinase capable of homo- and heterointeractions. Mol Cell Biol 34:965-977.

Stepp MA, Zieske JD, Trinkaus-Randall V, Kyne BM, Pal-Ghosh S, Tadvalkar G, and Pajoohesh-Ganji A (2014) Wounding the cornea to learn how it heals. Exp Eye Res 121:178-193.

Threadgill DW, Dlugosz AA, Hansen LA, Tennenbaum T, Lichti U, Yee D, LaMantia C, Mourton T, Herrup K, Harris RC, et al. (1995) Targeted disruption of mouse EGF receptor: effect of genetic background on mutant phenotype. Science 269:230-234.

Vedham V, Phee H, and Coggeshall KM (2005) Vav activation and function as a rac guanine nucleotide exchange factor in macrophage colony-stimulating factorinduced macrophage chemotaxis. Mol Cell Biol 25:4211-4220.

Wang YN and Hung MC (2012) Nuclear functions and subcellular trafficking mechanisms of the epidermal growth factor receptor family. Cell Biosci 2:13.

Watanabe T, Shintani A, Nakata M, Shing Y, Folkman J, Igarashi K, and Sasada R (1994) Recombinant human betacellulin. Molecular structure, biological activities, and receptor interaction. J Biol Chem 269:9966-9973.

Wieduwilt MJ and Moasser MM (2008) The epidermal growth factor receptor family: biology driving targeted therapeutics. Cell Mol Life Sci 65:1566-1584.

Wiley HS (2003) Trafficking of the ErbB receptors and its influence on signaling. Exp Cell Res 284:78-88.

Yamashiro DJ and Maxfield FR (1987) Acidification of morphologically distinct endosomes in mutant and wild-type Chinese hamster ovary cells. J Cell Biol 105:2723-2733.

Yokoyama M, Funatomi H, Kobrin M, Ebert M, Friess H, Buchler M, and Korc M (1995) Betacellulin, a member of the epidermal growth-factor family, is overexpressed in human pancreatic-cancer. Int J Oncol 7:825-829.

Zidar DA, Violin JD, Whalen EJ, and Lefkowitz RJ (2009) Selective engagement of $\mathrm{G}$ protein coupled receptor kinases (GRKs) encodes distinct functions of biased ligands. Proc Natl Acad Sci USA 106:9649-9654.

Address correspondence to: Brian P. Ceresa, University of Louisville, CTRB Room 305, 505 South Hancock Street, Louisville, KY 40202. E-mail: brian.ceresa@louisville.edu 